\title{
Semantically Congruent Visual Information Can Improve Auditory Recognition Memory in Older Adults
}

\section{Heikkilä, Jenni}

2018

Heikkilä , J , Fagerlund , P \& Tiippana , K 2018 , ' Semantically Congruent Visual Information Can Improve Auditory Recognition Memory in Older Adults ', Multisensory research , vol. 31 , no. 3-4 , pp. 213-225 . https://doi.org/10.1163/22134808-00002602

http://hdl.handle.net/10138/325651

https://doi.org/10.1163/22134808-00002602

unspecified

acceptedVersion

Downloaded from Helda, University of Helsinki institutional repository.

This is an electronic reprint of the original article.

This reprint may differ from the original in pagination and typographic detail.

Please cite the original version. 
Semantically congruent visual information can improve auditory recognition memory in older adults

Jenni Heikkiläㅁำ Petra Fagerlund ${ }^{2}$, Kaisa Tiippana ${ }^{1}$

${ }^{1}$ Department of Psychology and Logopedics, Faculty of Medicine, University of Helsinki.

${ }^{2}$ Department of Neuroscience and Biomedical Engineering, School of Science, Aalto University

Reference:

Heikkilä, J., Fagerlund P., Tiippana, K. (2018). Semantically congruent visual information can improve auditory recognition memory in older adults. Multisensory Research, 3-4, 213-225.

Author note:

The Multimodal Stimulus Set was developed by T.R. Schneider, S. Debener and A.K. Engel at the Dept. of Neurophysiology and Pathophysiology, University Medical Center Hamburg-Eppendorf, Germany.

Correspondence concerning this article should be addressed to:

Jenni Heikkilä

Department of Psychology and Logopedics, Faculty of Medicine, University of Helsinki. P.O. Box 9, 00014 University of Helsinki, Finland.

E-mail: jenni.heikkila@helsinki.fi 


\begin{abstract}
In the course of normal aging, memory functions show signs of impairment. Studies of memory in the elderly have previously focused on a single sensory modality, although multisensory encoding has been shown to improve memory performance in children and young adults. In this study, we investigated how audiovisual encoding affects auditory recognition memory in older (mean age 71 years) and younger (mean age 23 years) adults. Participants memorized auditory stimuli (sounds, spoken words) presented either alone or with semantically congruent visual stimuli (pictures, text) during encoding. Subsequent recognition memory performance of auditory stimuli was better for stimuli initially presented together with visual stimuli than for auditory stimuli presented alone during encoding. This facilitation was observed both in older and younger participants, while the overall memory performance was poorer in older participants. However, the pattern of facilitation was influenced by age. When encoding spoken words, the gain was greater for older adults. When encoding sounds, the gain was greater for younger adults. These findings show that semantically congruent audiovisual encoding improves memory performance in late adulthood, particularly for auditory verbal material.
\end{abstract}

Keywords: audiovisual, aging, memory, semantic congruency 


\section{Introduction}

Aging leads to decreases in memory performance. An extremely important question is how memory encoding can be improved in order to help seniors to compensate for their declining memory. The aim of this study was to investigate whether semantically congruent audiovisual information can enhance memory encoding in older adults, and if so, whether younger and older adults differ in how they utilize multisensory information in encoding.

Age-related decline in memory functions

In the course of normal aging, some memory functions decline, while others show no signs of impairment (see Old and Naveh-Benjamin, 2008, for a review). Working memory and short term memory deteriorate with age (Bopp and Verhaeghen, 2005; Park et al., 2002). Studies of long-term memory show that episodic memory (memory of experiences and specific events in time in a serial form, such as recall, recognition or source memory) and semantic memory (knowledge memory) decline in older age, but at different rates. Episodic memory, measured e.g. by recall of word lists, starts to decline around the age of 60 years, while semantic memory, measured e.g. by vocabulary and general knowledge, increases until 55 years and starts to decline around 65 years of age (Rönnlund, Nyberg et al., 2005). Older adults (66-84 years) perform less accurately than younger adults (18-22 years) in episodic recognition memory tasks (where the task is to remember words) but there is no difference between the age groups in semantic recognition memory tasks (Spaniol, Madden \& Voss, 2006). Taken together, episodic memory declines during normal aging.

Episodic memory includes memory encoding, retention and retrieval processes. The encoding process, especially intentional encoding and learning, is impaired in old age (Old and NavehBenjamin, 2008). The decline of memory encoding may be reflected in recognition memory tasks. Grady et al. (1995) showed that recognition memory of faces is impaired in older adults (mean age 
69 years) compared to younger adults (25 years), and that the medial temporal cortex, including the hippocampus, is activated during encoding in younger, but not in older adults. The authors proposed that the age-related impairment of recognition memory may be due to inadequate encoding of stimuli. Encoding is affected by many factors, including the sensory modalities used to receive and process the incoming information and its semantic content.

\section{Multisensory semantic congruency facilitates memory performance}

There is accumulating evidence that episodic memory can be improved by semantically congruent multisensory stimuli in younger adults. When stimuli are encoded in a semantically congruent audiovisual context, later recognition memory performance improves. For example, the meowing of a cat is remembered better if presented with a picture of a cat during encoding, rather than alone or with a meaningless picture. This audiovisual congruency effect has been shown to exist for several stimulus combinations in young adults (Murray et al., 2004; Lehmann and Murray, 2005; Moran et al., 2013; Thelen et al., 2015; Ueno et al., 2015; Heikkilä et al., 2015, 2017a,b, but see Cohen et al., 2009) and in children (Heikkilä and Tiippana, 2016).

However, we are aware of only one previous study on audiovisual memory encoding in older adults. Luo, Hendriks and Craik (2007) found that when written words were presented with congruent sounds during encoding, only younger adults benefited from the sounds. For older adults, recognition memory performance was not affected by whether the written words were initially presented alone or with sounds. Therefore, memory for written words presented with sounds during encoding does not have the same benefit for older adults as in younger adults, but it is not known whether possible multisensory memory benefits exist for other audiovisual stimulus combinations.

We hypothesized that older adults could benefit from congruent audiovisual information in memory encoding, such as has been shown previously for younger adults. In the current study, we used the 
same paradigm as in Heikkilä et al. (2017b), but with older adults as participants, and therefore we describe it in detail here. In Heikkilä et al. (2017b), we studied the audiovisual congruency effect using an experimental paradigm, which enabled us to exclude possible interference by incongruent or non-semantic stimuli. Young adult participants memorized sounds, spoken words or written words, each of which was presented with either a semantically congruent stimulus in the other modality or alone during encoding. The semantically congruent audiovisual stimulus pairs and unisensory stimuli were presented in separate blocks. This design allowed us to compare the precision of recognition memory between congruent audiovisual and unisensory situations using the discriminability index $d^{\prime}$. The $d^{\prime}$ is a measure that reflects the observer's ability to discern previously presented, memorized items from new ones (for a review, see Murdock, 1982). It precludes the possible influence of criterion shifts on the congruency effect. The recognition memory of sounds was better when initially presented with semantically congruent pictures or written words than when presented alone. The recognition memory of spoken words was facilitated by semantically congruent pictures. Written words did not facilitate memory encoding of spoken words and vice versa. These results show that semantically congruent audiovisual stimuli enhance auditory memory performance in young adults when verbal and non-verbal stimuli are presented together during encoding.

\section{Multisensory perception in older adults}

Even though multisensory memory enhancement has not yet been reported in older adults, it could be expected to exist on the basis of findings regarding multisensory perception during aging. Several studies have reported that multisensory integration increases in older adults (Laurienti et al., 2006; Peiffer et al., 2007; Diedrich et al., 2008). Older adults show more visual influence in speech perception than younger adults, i.e. they use information from the talking face more efficiently to support auditory speech comprehension (Cienkowski and Carney, 2002; Sekiyama, Soshi \& 
Sakamoto, 2014; Setti et al., 2013; Stevenson et al., 2015; Winneke and Phillips, 2011), even though sometimes no difference is reported (e.g. Sommers, Tye-Murray \& Spehar, 2005). Meaningful context can contribute to audiovisual speech perception in older and younger adults (Tye-Murray et al., 2008; Gordon and Allen, 2009; Maguinness et al., 2011). Maguinness et al. (2011) point out that that visual speech may benefit older adults particularly when the semantic content of the speech is unpredictable (such as meaningless strings of words).

Multisensory perception is thus resistive to aging, and may sometimes even be enhanced in older age. Better perceptual processing or stimulus recognition is generally reflected into improved memory encoding. Consequently, it could be hypothesized that the preserved ability to perceive multisensory stimuli in aging might be reflected into multisensory memory benefits. For example, audiovisual semantic congruency could be assumed to improve recognition memory performance in older age.

\section{Current study}

Our aim was to study the benefits of audiovisual semantic congruency in memory encoding in older adults. We chose to investigate the effect of visual stimuli on auditory recognition memory performance since visual information has been shown to be beneficial when encoding auditory information (Moran et al., 2013; Heikkilä et al., 2015, 2017b; Thelen et al., 2015). This is in line with the finding that semantic visual information influences auditory object recognition more than vice versa (Yuval-Greenberg and Deouell, 2009). In auditory tasks, visual stimuli influence performance more in older than younger adults (Van Gerven and Guerreiro, 2016). Diaconescu et al. (2013) found that both younger and older adults are faster to respond to sounds when they are presented with pictures than vice versa. However, older adults show a larger reaction time gain in audiovisual conditions compared to younger adults. If these findings are reflected into memory encoding, 
congruent visual stimuli could be expected to affect auditory encoding in older adults at least to a similar extent as in younger adults.

The present study applied an experimental design in which semantically congruent audiovisual stimulus pairs or auditory stimuli were presented. In contrast to our earlier studies (Heikkilä et al., 2015; Heikkilä and Tiippana, 2016), we did not include incongruent or non-semantic audiovisual stimuli to avoid possible interference from them (see Thelen et al., 2015). Also, this design allows to compare the precision of recognition memory $\left(d^{\prime}\right)$ between congruent audiovisual and auditory situations (Heikkilä et al., 2017b).

We studied the effect of semantically congruent pictures and written words on the recognition memory of sounds and spoken words. Our hypothesis was that older adults benefit from multisensory information during memory encoding similarly as younger adults because multisensory perception does not decline with age (Laurienti et al., 2006; Peiffer et al., 2007; Diedrich et al., 2008).

\section{Methods}

\section{Participants}

The participants in this study were 42 older adults (mean age 71 years, SD $4.4 ; 15$ males). We compared their results with 42 young adults (mean age 23 years, SD 3.5; 9 males), whose results have been published in Heikkilä et al. (2017b). Participants were native speakers of Finnish, had normal or corrected to normal vision and normal hearing. They reported no problems in memory or any 
neurological illnesses or learning difficulties. The research has received ethical approval from the University of Helsinki Review Board in the Humanities and Social and Behavioural Sciences.

\section{Stimuli}

Sounds of natural objects, pictures of natural objects, spoken words and written words were used as stimulus material. These stimuli were also used in our previous studies (Heikkilä et al., 2015; 2017a,b; Heikkilä \& Tiippana, 2016).

The sounds were recordings of complex sounds of objects from several semantic categories (animals, tools, vehicles, musical instruments, and household items). They were obtained from the Multimodal Stimulus Set (Schneider et al., 2008) or from the internet and edited to resemble the sounds obtained from the Multimodal Stimulus Set. The sounds were edited to have a duration of $400 \mathrm{~ms}$. The spoken words were common one or two-syllable Finnish nouns from different semantic categories (animals, tools, foods, plants and household items etc.). They were spoken by a female speaker, and their duration varied between 350 and $780 \mathrm{~ms}$. The intensities of all auditory stimuli were equalized by equalizing the root mean square power. The auditory stimuli were presented at approximately 50 $\mathrm{dB}(\mathrm{A})$.

The pictures were photographs selected from the Multimodal Stimulus Set (Schneider, Engel \& Debner, 2008) or gathered from the internet and modified to resemble those in the Multimodal Stimulus Set. The photographs presented objects from different semantic categories (animals, tools, vehicles, musical instruments, and household items, see Schneider, Engel \& Debner, 2008 for details). Photographs were converted into gray scale and presented on a black background. They were positioned centrally on a computer monitor. Their sizes varied between 1 and 20 degrees horizontally and vertically. The written words were common one or two-syllable Finnish nouns from various semantic categories (animals, tools, foods, plants, household items etc.). They were presented in white 
Times New Roman in the center of the black computer monitor. The size was 0.57 degrees. The visual stimuli were presented either for $400 \mathrm{~ms}$ (with sounds) or $800 \mathrm{~ms}$ (with spoken words).

\section{Design and procedure}

The same experimental conditions were used in our previous study (Heikkilä et al., 2017b), in which the results of younger participants were originally published. The results of older participants are new and previously unpublished.

The experiment consisted of auditory and audiovisual conditions presented to the participants in random order. Each condition consisted of two phases: an encoding phase followed by a recognition memory phase. There was a break of ca. 2 min between the conditions.

In auditory conditions, only auditory stimuli were presented in the encoding phase and the participants were instructed to memorize the presented stimuli. In audiovisual conditions, during the encoding phase, audiovisual stimulus pairs were presented. The items with the pair were always semantically congruent: for example, a spoken word "mouse" and a picture of a mouse, or the same word spoken and written. The audiovisual items were presented with simultaneous onsets of their components. The participants were instructed to memorize the auditory stimuli. During the $800 \mathrm{~ms}$ inter-stimulus interval in the encoding phase, a black screen with a white fixation cross in the center was presented.

The recognition memory phase immediately followed the encoding phase, and it was always auditory. Here only the memorized auditory stimuli were presented again, intermingled with an equal number of new stimuli. The participant's task was to decide for each stimulus whether or not it had been presented in the encoding phase by pressing one of two keys in a response box. The next item was presented $1000 \mathrm{~ms}$ after the response. 
The experiment was conducted in a quiet room. The auditory stimuli were presented binaurally through headphones. The visual stimuli appeared on a computer screen. The stimuli were presented and the response data gathered with Presentation software (version 16.3, Neurobehavioral systems), which is a stimulus delivery and experiment control program for neuroscience. The order of stimuli was always random. Before the experiment, the participants read written instructions and performed a short practice session. During the experiment, the experimenter sat next to the participant to ensure that the participant maintained his or her gaze on the screen.

\section{Memory of sounds}

The effect of semantically congruent pictures and written words on the recognition memory of sounds was studied with three encoding conditions: one auditory and two audiovisual. In the encoding phase of the auditory condition, sounds were presented alone. In the encoding phase of one audiovisual condition, sounds were paired with congruent pictures, and in the other audiovisual condition, sounds were paired with congruent written words. There were 25 sounds in each condition. The participants ` task was to memorize the sounds.

\section{Memory of spoken words}

The effect of semantically congruent pictures and written words on the recognition memory of spoken words was studied with three encoding conditions: one auditory and two audiovisual tasks. In the encoding phase of the auditory condition, spoken words were presented alone. In the encoding phase of one audiovisual condition, spoken words were paired with congruent pictures, and in the other audiovisual condition, spoken words were paired with congruent written words. There were 51 sounds in each condition. The participants` task was to memorize the spoken words. 


\section{Data analysis}

In the analyses, we utilized $d^{\prime}$ (Green \& Swets, 1966; Maximillian \& Creelmann, 1991; Stanislav \& Todorov, 1999, see also Heikkilä et al., 2017b). In our recognition memory paradigm, $d^{\prime}$ reflects the participant's ability to discriminate between two stimulus classes: memorized and new. $d^{\prime}$ was calculated using the hit rate and the false alarm rate of each recognition memory task. Hits were those trials in which the participant recognized the memorized items correctly (correct responses; old identified as old). False alarms $(F A)$ were those trials in which participant recognized new items as previously presented (new recognized as old). These values were then normalized to obtain the $z$ score values $z(H I T)$ and $z(F A) \cdot d^{\prime}$ was calculated by subtracting them from one another: $d^{\prime}=z(H I T)$ $z(F A)$. The extreme rates, i.e. false alarm rates of 0 were corrected using $1 / 2 \mathrm{n}$, and hit rates of 1 were corrected using $1-1 / 2 \mathrm{n}$, where $\mathrm{n}$ is the number of trials (Miller, 1996). The $d^{\prime}$ :s were analyzed with a mixed model analysis of variance (ANOVA) with Modality (auditory, with pictures, with text), Stimulus type (sounds, spoken words) and Age (younger, older) as factors. Greenhouse-Geisser correction was applied to $\mathrm{p}$ values when appropriate. However, the original degrees of freedom are reported. Partial eta squared $\left(\eta^{2}\right)$ are reported. 


\section{Results}

The recognition memory performance $\left(d^{\prime}\right)$ for sounds and spoken words presented with visual stimuli or alone during encoding is presented in Figure 1.

YOUNGER ADULTS
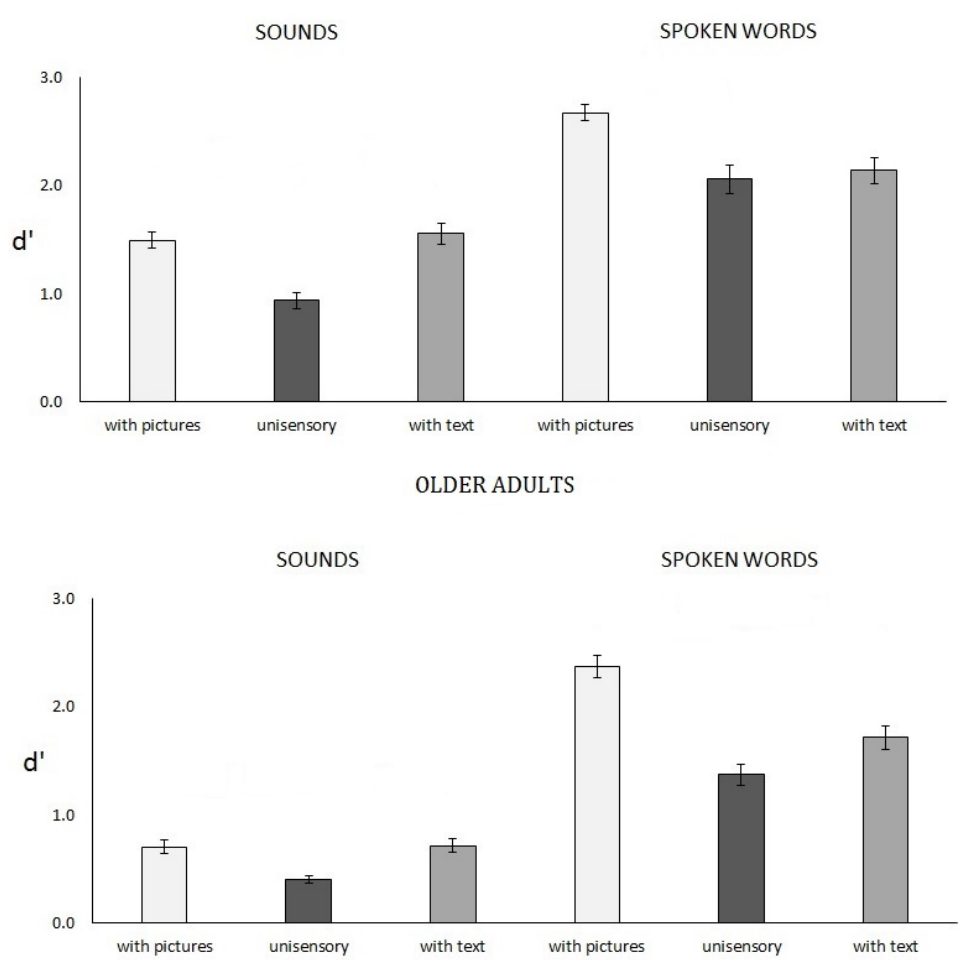

Figure 1. Recognition memory performance $\left(d^{\prime}\right)$ for sounds and spoken words presented alone or together with semantically congruent pictures or written words during encoding in younger and older adults. Error bars represent standard error of the mean.

A mixed models ANOVA was conducted with Modality (auditory, with pictures, with text), Stimulus type (sounds, spoken words) and Age (younger, older) as factors. The main effects of Modality $\left[F(2,164)=70.9, p<.0001, \eta^{2}=.464\right]$, Stimulus type $\left[F(1,82)=271.4, p<.0001, \eta^{2}=.768\right]$ and Age 
$\left[F(1,82)=360.6, p<.0001, \eta^{2}=.425\right]$ were significant, as were the interactions between Modality and Stimulus type $\left[F(2,164)=21.1, p<.0001, \eta^{2}=.205\right]$ and Modality, Stimulus type and Age $\left[F(2,164)=6.3, p=.003, \eta^{2}=.072\right]$. Memory performance was better when the memorized stimulus was paired with either a picture or text compared to auditory presentation. Performance was superior with pictures compared to text. Spoken words were remembered better than sounds. Memory performance was better in younger than in older adults.

The congruency effect was further investigated by studying the interaction between Modality, Stimulus type and Age with gain indices. The gain index was calculated as the difference between audiovisual and auditory memory performance (audiovisual $d^{\prime}$ minus auditory $d^{\prime}$ ). The gain index was calculated for each participant and audiovisual condition (sounds with pictures; sounds with text; spoken words with pictures; spoken words with text). The gain indices describe the relative amount of memory enhancement for stimuli encoded in audiovisual vs. auditory context. They were studied using an ANOVA with Modality (gain due to pictures, gain due to text), Stimulus type (sounds, spoken words) and Age (younger, older) as factors. The main effect of Modality was significant $\left[F(1,82)=25.6, p<.0001, \eta^{2}=.238\right]$, as were the interactions between Modality and Stimulus type $\left[F(1,82)=36.6, p<.0001, \eta^{2}=.309\right]$ and Stimulus type and Age $\left[F(1,82)=14.6, p<.0001, \eta^{2}=.151\right]$. The main effect of Modality suggested that pictures produced a larger memory gain than text. However, post-hoc tests for the interaction between Modality and Stimulus type showed that this difference was significant only for spoken words $[\mathrm{t}(83)=7.39, \mathrm{p}<.0001]$, not for sounds $[\mathrm{t}(83)=-.512, \mathrm{p}=.610]$.

In order to study the interaction between Stimulus type and Age, the gain indices were combined across modality (gain for sounds with pictures plus gain for sounds with text; gain for spoken words with pictures plus gain for spoken words with text), and these combined gain indices (Fig. 2) were studied using an ANOVA with Stimulus type (gain for sounds, gain for spoken words) and Age (younger, older) as factors. When encoding sounds, younger adults benefited more from audiovisual presentation than older adults $\left[F(1,82)=7.19, p=.009, \eta^{2}=.081\right]$. When encoding spoken words, older 
adults benefited more from audiovisual presentation than younger adults $[F(1,82)=6.65, p=.012$, $\left.\eta^{2}=.075\right]$

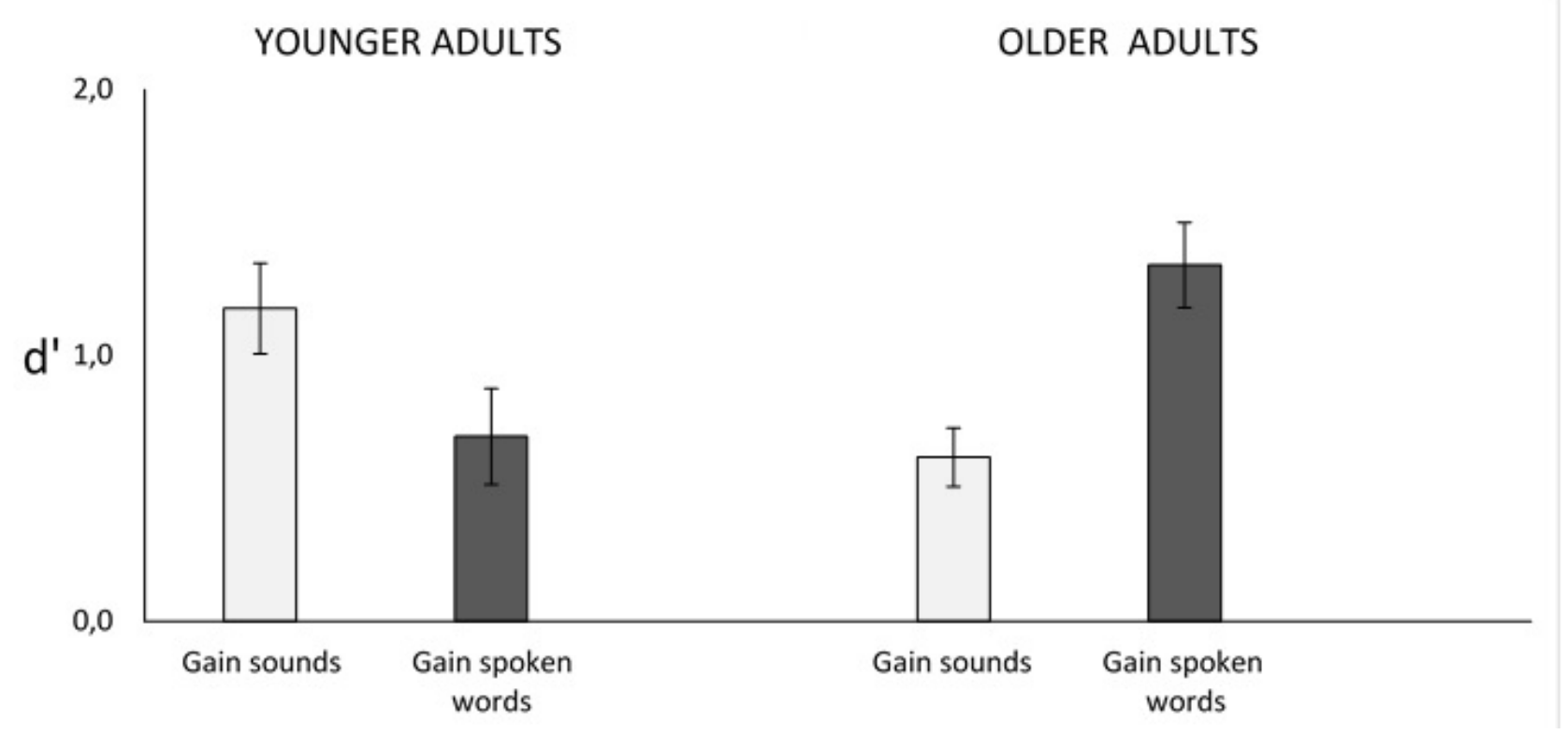

Figure 2. Combined gain indices for sounds and spoken words in younger and older adults. Error bars represent standard error of the mean.

\section{Discussion}

The results of the present study show that the semantic congruency of multisensory stimuli can improve the recognition memory performance in older adults. When auditory stimuli were paired with semantically congruent visual stimuli during encoding, memory was better than with only auditory presentation. This facilitation was observed in both older and younger adults. Thus, not only younger but also older adults can utilize multisensory information to enhance their memory performance. This is the first study to show that older adults can benefit from multisensory memory 
cues. These findings are in line with previous studies conducted with young adults (Murray et al., 2004; Lehmann and Murray, 2005; Moran et al., 2013; Heikkilä et al., 2015, 2017a,b; Thelen et al., 2015; Ueno et al., 2015) and with children (Heikkilä and Tiippana, 2016).

There were differences in how younger and older adults utilized audiovisual information in memory encoding. The overall memory performance was poorer in older adults compared to younger, in line with previous findings (Old and Naveh-Benjamin, 2008). When this baseline difference was accounted for by using gain indices, older adults benefited more from multisensory presentation than younger adults when encoding spoken words. However, when encoding sounds, younger adults benefited more from multisensory presentation than older adults.

The gain from semantically congruent visual stimuli was greater for older than younger participants when spoken words were memorized. Older adults utilize audiovisual speech, especially when listening conditions are challenging (e.g. Tye-Murray et al., 2008). They benefit more from the presence of visual speech than younger adults when the task is to recall meaningless sentences (Maguinness et al., 2011). It may be that not only visual speech but also other semantically congruent visual information (pictures or written words) can help older adults to resolve the phonetic and semantic information in unpredictable speech, i.e. a random sequence of unrelated spoken words, thus enhancing the memory encoding relatively more than for younger adults.

The recognition memory of natural sounds of objects and events was improved by pictures and written words in both age groups. However, younger adults benefited more from multisensory presentation than older adults. Their overall memory performance was also better. It may be that older adults had trouble processing briefly presented stimuli because of the slowing down of general information processing (Salthouse, 1996; 2000). The stimulus presentation rate in conditions including sounds was quite fast (400 ms/stimulus). It may have been too fast for older adults for efficient information processing. This may have led to poorer encoding of both auditory and visual information, the former 
resulting in lower overall memory level for sounds, and the latter in smaller audiovisual gain in older adults.

In general, we propose that the presentation of semantically congruent visual stimuli facilitated the recognition of the to-be-memorized auditory stimuli, which improved their encoding in memory. Another possibly contributing factor may be attentional enhancement if multisensory items capture attention more than unisensory items (Talsma, Senkowski, Soto-Faraco \& Woldorff, 2010). However, in our previous studies (Heikkilä et al., 2015; Heikkilä \& Tiippana, 2016; Heikkilä, Alho \& Tiippana, 2017a), we have controlled for the alerting/distracting effect of multisensory stimuli by using nonsemantic and incongruent audiovisual stimulus pairs in addition to congruent ones, so that all stimuli during encoding were audiovisual. In these studies, we also observed the congruency effect, so that it is not solely due to attentional enhancement.

Taken together, the results of this study show that semantically congruent multisensory experiences during encoding can improve memory performance during normal aging. This finding opens new, interesting avenues for future research on the benefits of multisensory memory and learning in later life, which we predict will be a growing topic, with potential practical relevance in normal aging and age-related memory impairment. 


\section{References}

Bopp, K. \& Verhaeghen, P. (2005). Aging and verbal memory span: A meta-analysis. Journal of Gerontology: Psychological Sciences, 60b, 223-33.

Cienkowski, K. and Carney, A. (2002). Audio-visual speech perception and aging. Ear and Hearing, $23,439-49$.

Cohen, M. A., Horowitz, T. S., \& Wolfe, J. M. (2009). Auditory recognition memory is inferior to visual recognition memory. Proceedings of National Academy of Science U S A, 106(14), 6008-6010.

Diaconescu, A., Hasher, L. \& McIntosh, A. (2013). Visual dominance and multisensory integration changes with age. NeuroImage, 65,152-66.

Diederich, A., Colonius, H. \& Schomburg, A. (2008). Assessing age-related multisensory enhancement with the time-window-of -integration model. Neuropsychologia, 46,2556-62.

Gordon, M. S., \& Allen, S. (2009). Audiovisual speech in older and younger adults: Integrating a distorted visual signal with speech in noise. Experimental Aging Research, 35(2), 202-219.

Grady, C., McInthos, A., Horwitz, B., Maisog, J., Ungerleider, L., Mentis, M., Pietrini, P., Schapiro, M. \& Haxby, J. (1995). Age-related reductions in human recognition memory due to impaired encoding. Science, 269.

Green, D.M. \& Swets, J. (1966). Signal detection theory and psychophysics. New York: Wiley.

Heikkilä, J., Alho, K., Hyvönen, H. \& Tiippana, K. (2015). Audiovisual semantic congruency during encoding enhances memory performance. Experimental Psychology, 62,123-30.

Heikkilä, J., Alho, K. \& Tiippana, K. (2017a). Semantic congruency improves recognition memory performance for both audiovisual and visual stimuli. Accepted to publication, Multisensory Research.

Heikkilä, J., Alho, K. \& Tiippana, K. (2017b). Semantically congruent visual stimuli can improve auditory memory. In press, Multisensory Research. 
Heikkilä, J. \& Tiippana, K. (2016). School-aged children can benefit from audiovisual semantic congruency during memory encoding. Experimental Brain Research, 234, 1199-1207.

Laurienti, P.J., Burdette, J.H., Maldjian, J.A., \& Wallace, M.T. (2006). Enhanced multisensory integration in older adults. Neurobiology of Aging, 27,1155-563

Lehmann, S. \& Murray, M.M. (2005). The role of multisensory memories in unisensory object discrimination. Cognitive Brain Research, 24, 326-34.

Luo, L. Hendriks, T. and Craik, F. (2007). Age differences in recollection: Three patterns of enhanced encoding. Psychology and Aging, 22, 269-80.

Macmillan, N \& Creelman, C. (1991). Detection theory: A user's guide. New York: Cambridge University Press.

Maguinness, C., Setti, A., Burke, K., Kenny, R. A., \& Newell, F. (2011). The effect of combined sensory and semantic components on audio-visual speech perception in older adults. Frontiers in Aging Neuroscience, 3, 19.

Miller, J. (1996). The sampling distribution of d'. Perception and Psychophysics, 58, 65-72.

Moran, Z., Bachman, P., Pham, P., Cho, S., Cannon, T. \& Shams, L. (2013). Multisensory encoding improves auditory recognition. Multisensory Research, 26,581-92.

Murdock, B. (1982). Recognition memory. In Puff, C.R. Handbook of Research Methods in Human Memory and Cognition. Academic Press Inc. London, UK.

Murray, M.M., Michel, C.M., Grave de Peralta, R., Ortigue, S., Brunet, D., Gonzalez Andino, S. \& Schnider, A. (2004). Rapid discrimination of visual and multisensory memories revealed by electrical neuroimaging. Neurolmage, 21, 125-35.

Old, S. \& Naveh-Benjamin, M. (2008). Age-related changes in memory. In Hofer, S. \& Alwin, D. (eds.) Handbook of Cognitive Aging. 2008, UK, Sage Publications.

Park, D., Lautenschlager, G., Hedden, T., Davidson, N., Smith, A. \& Smith, P. (2002). Models of visuospatial and verbal memory across the adult life span. Psychology and Aging, 17,826-37. 
Peiffer,A., Mozolic, J., Hugenschmidt, C. \& Laurienti, P. (2007). Age-related multisensory enhancement in a simple audiovisual detection task. NeuroReport, 18,1077-81

Rönnlund, M., Nyberg, L., Bäckman, L. \& Nilsson, L. (2005). Stability, Growth, and Decline in Adult Life Span Development of Declarative Memory: Cross-Sectional and Longitudinal Data From a Population-Based Study. Psyhology and Aging, 20,3-18.

Salthouse, T. (1996). The processing speed theory of adult age differences in cognition. Psychological Review, 103,403-28.

Salthouse, T. (2000). Aging and measures of processing speed. Biological Psychology, 54,35-54.

Schneider, T.R., Engel, A.K. \& Debener, S. (2008). Multisensory identification of natural objects in a two-way crossmodal priming paradigm. Experimental Psychology, 55, 121-32.

Sekiyama, K., Soshi, T., \& Sakamoto, S. (2014). Enhanced audiovisual integration with aging in speech perception: a heightened McGurk effect in older adults. Frontiers in Psychology, 5, 323.

Setti, A., Burke, K. E., Kenny, R., \& Newell, F. N. (2013). Susceptibility to a multisensory speech illusion in older persons is driven by perceptual processes. Frontiers in Psychology, 4, 575.

Sommers, N Tye-Murray, B Spehar (2005). Auditory-visual speech perception and auditory-visual enhancement in normal hearing younger and older adults. Ear and Hearing, 26,263-75.

Spaniol, J., Madden, D. \& Voss, A. (2006). A diffusion model analysis of adult age differences in episodic and semantic long-term memory retrieval. Journal of Experimental Psychology: Learning, Memory and Cognition, 32,101-17.

Stanislav, H. \& Torodov, N. (1999). Calculating signal detection theory measures. Behavior Research Methods, Instruments and Computers, 31, 137-49.

Stevenson, R. A., Nelms, C. E., Baum, S. H., Zurkovsky, L., Barense, M. D., Newhouse, P. A., \& Wallace, M. T. (2015). Deficits in audiovisual speech perception in normal aging emerge at the level of whole-word recognition. Neurobiology of Aging, 36(1), 283-291.

Talsma, D., Senkowski, D., Soto-Faraco, S., \& Woldorff, M. G. (2010). The multifaceted interplay between attention and multisensory integration. Trends in Cognitive Science, 14(9), 400-410. 
Thelen, A., Talsma, D. \& Murray, M. (2015). Single-trial multisensory memories affect later auditory and visual object discrimination. Cognition, 138, 148-60.

Tye-Murray, N., Sommers, M., Spehar, B., Myerson, J., Hale, S., \& Rose, N. S. (2008). Auditoryvisual discourse comprehension by older and young adults in favorable and unfavorable conditions. Int J Audiol, 47(sup2), S31-S37.

Ueno, D., Masumoto, K., Sutani, K. \& Iwaki, S. (2015). Latency of modality-specific reactivation of auditory and visual information during episodic memory retrieval. NeuroReport, 26, 302-8.

Winneke, A. H., \& Phillips, N. A. (2011). Does audiovisual speech offer a fountain of youth for old ears? An event-related brain potential study of age differences in audiovisual speech perception. Psychology of Aging, 26(2), 427-438.

Yuval-Greenberg, S., \& Deouell, L. Y. (2009). The dog's meow: asymmetrical interaction in crossmodal object recognition. Experimental Brain Research, 193(4), 603-614.

Van Gerven, P. \& Guerreiro, M. (2016). Selective attention and sensory modality in aging: curses and blessings. Frontiers in Human Neuroscience, 10,147. 